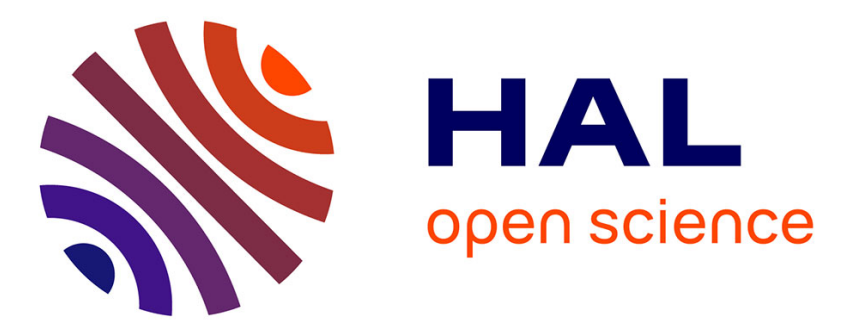

\title{
Proposed architecture for integrity monitoring of a GNSS/MEMS system with a Fisheye camera in urban environment
}

Enik Shytermeja, Axel Javier Garcia Peña, Olivier Julien

\section{To cite this version:}

Enik Shytermeja, Axel Javier Garcia Peña, Olivier Julien. Proposed architecture for integrity monitoring of a GNSS/MEMS system with a Fisheye camera in urban environment. ICL-GNSS 2014, IEEE International Conference on Localization and Global Navigation Satellite Systems, IEEE, Jun 2014, Helsinki, Finland. 10.1109/ICL-GNSS.2014.6934179 . hal-01135154

\section{HAL Id: hal-01135154 \\ https://hal-enac.archives-ouvertes.fr/hal-01135154}

Submitted on 12 May 2015

HAL is a multi-disciplinary open access archive for the deposit and dissemination of scientific research documents, whether they are published or not. The documents may come from teaching and research institutions in France or abroad, or from public or private research centers.
L'archive ouverte pluridisciplinaire HAL, est destinée au dépôt et à la diffusion de documents scientifiques de niveau recherche, publiés ou non, émanant des établissements d'enseignement et de recherche français ou étrangers, des laboratoires publics ou privés. 


\title{
Proposed Architecture for Integrity Monitoring of a GNSS/MEMS System with a Fisheye Camera in Urban Environment
}

\author{
Enik Shytermeja, Axel Garcia-Pena, Olivier Julien \\ TELECOM/SIGNAV Lab, ENAC, Toulouse, France \\ Email : shytermeja@recherche.enac, garcia-pena@recherche.enac.fr, ojulien@recherche.enac.fr
}

\begin{abstract}
Recently, an increasing trend of GNSS-based Safety Critical (SC) or Liability Critical (LC) urban applications has been observed. These applications, associated to dense urban canyons, can have very stringent requirements in terms of accuracy, reliability and availability of the provided position solution. In this paper, the implementation of a step-by-step algorithm able to ensure the robustness and integrity monitoring of an integrated GPS/Galileo receiver with low-cost MEMS sensors and aided by the video Fisheye camera is proposed.
\end{abstract}

Keywords-component: GNSS, VDFLL, MEMS, Integrity monitoring, Video Fisheye, IBPL, RAIM, EKF, urban environment.

\section{INTRODUCTION}

In the last decade, Global Navigation Satellites Systems (GNSS) have gained a significant position in the development of Urban Navigation applications and associated services. A major concern of the constant growth of GNSS-based urban applications is related to the quality of the positioning service, expressed in terms of accuracy, availability and continuity of service but also of integrity provision, ensuring that the application requirements are met [6].

Recently, a great attention of the European Commission (EC) and European GNSS Agency (GSA) has been given to the development of several research projects focused on the road transportation sector, as in [1], [2]. The European Road Federation Union (ERF), based on the integrity requirement perspective, classifies road applications in two main categories, such as [3]: Safety of Life (SoL) applications, where the main representatives are the Advance Driver Assistance Systems (ADAS) and Emergency Services Management (ESM) and Liability Critical (LC) applications including Road User Charging (RUC), Pay per use insurance pricing, On street parking pricing and so on. However, the integrity monitoring concept in Road User applications, was firstly introduced and developed within the framework of Liability Critical applications, where Electronic Toll Collection (ETC) constitutes the main representative. In ETC systems, an erroneous positioning leads to an incorrect user charging. Therefore, in order to meet the integrity requirements, GNSS has been recognized as the most flexible and cost-effective technology to be embedded in ETC systems [1].

In dense urban environments, such as city centers, traditional GNSS signal processing techniques are incapable of providing an accurate and reliable position solution due to the frequent blockage of the Line-Of-Sight (LOS) signals and the presence of diffractions/reflections of the transmitted signal (multipath). These effects severely affect the pseudo-range and Doppler measurements, used by a GNSS receiver for the position computation. Therefore, advanced GNSS signal processing techniques are necessary to mitigate the undesired effects introduced from the urban environment. It is thus preferable to go toward a multi-constellation GNSS (GPS + Galileo) receiver for measurements' redundancy, coupled with the use of complementary sensors. However, this approach might only manage to provide an accurate positioning but still does not ensure a measure of trust of the positioning solution.

The concept of integrity is well known from the civil aviation sector, as being a Safety-of-Life (SoL) application, where very stringent performance requirements should be met along with the provisioning of timely warnings when the system should not be trusted. Several studies have addressed the problematic of integrity monitoring in urban environments [4], but neither proper methodologies nor urban user requirements and standards are publicly available in this field.

The aim of this work is to propose an innovative step-by-step approach that is capable of providing integrity monitoring of a hybridized GNSS/MEMS/Video system in urban canyons.

This paper is organized as follows: Section II describes the problematic of urban environment severely affecting the positioning performance while the detailed description of our proposed solution is given in Section III. Section IV provides the logic behind the choice of Vector Delay/Frequency Lock Loop (VDFLL). Then, Section V is dedicated to the mathematical error modeling of the inertial low-cost Micro-machined ElectroMechanical (MEMS) sensor. In Section VI, the Video Fisheye camera technique for GNSS NLOS signals rejection, is addressed. Section VII deals with the advanced multipath mitigation techniques while in Section VIII a comparison of the two candidate algorithms for integrity monitoring provision, is given. The main conclusions of this paper and future work will be drawn in Section IX and X, respectively.

\section{ThE PROBLEMATIC IN URBAN ENVIRONMENT}

The urban environment presents several challenges to GNSS signal reception, severely degrading the positioning accuracy. The main problems arising from the urban environment conditions, are the following: 
i. Multipath: is defined as the reception of reflected or diffracted echoes (from the ground, buildings, foliage, lampposts and so on) of the desired LOS GNSS signal.

ii. Attenuation or blockage of the GNSS LOS signal: is a phenomenon arising due to the partial or total obstruction of the GNSS LOS from the urban environment characteristics.

iii. Interference: occurring due to the presence of a wide class of interfering signals, falling within the GNSS frequency bands. The dominant source of interference is related to the reception of continuous wave interference (CWI) signals, generated from Ultra-High Frequency (UHF) and Very-High Frequency (VHF) TV transmitters, Digital Video Broadcasting Terrestrial (DVB-T) system and so on.

The consequences of the above mentioned urban environment error sources either on the received signal or at the receiver correlator output w.r.t. the ideal reception of a GNSS signal are given next. The mitigation of these consequences constitutes the initial step of the proposed solution.

a. Distortion of the receiver's correlation function: between the received multipath-contaminated signal and the receiver's locally generated replica. In the GNSS context, this is the main consequence of multipath, affecting the signal code and carrier tracking accuracies, and thus introducing large degradations to the pseudo-range accuracy and therefore producing an inaccurate position solution [5].

b. Only NLOS signals reception: occurs when the direct LOS GNSS signal is blocked and thus, only reflected signals are received. This results in a pseudo-range measurement error and consequently a decrease of the positioning accuracy.

c. Incongruent GNSS pseudo-range measurements: occurring when a set of pseudo-range measurements, provided from one or more GNSS satellites, are not congruent with the other ones. This event is the outcome of the three error sources mentioned above.

These severe urban conditions lead to large measurement errors and consequently to inaccurate positioning. In the worst case, the GNSS positioning capability can be unavailable. Therefore, it is evident that advanced signal processing techniques are necessary to mitigate these undesired effects in order to ensure the accuracy and availability of the position solution.

\section{PROPOSED SOLUTION}

To overcome the urban environment constraints, strongly affecting the positioning performance and to guarantee a satisfying level of trust of the PVT solution, the development of a novel step-by-step technique consisting of an integrated GPS/Galileo receiver + low-cost MEMS sensors, aided by a video Fisheye camera and implementing an urban-oriented integrity monitoring technique is proposed. Fig. 1 shows the schematic structure of the proposed technique for the provision of GNSS positioning and integrity monitoring in dense urban environment. The rationale for the algorithm lies on the implementation of different sub-techniques, each aiming at the mitigation of the three major error sources in urban environments, identified in section II. The starting point of the approach is the inaccurate position solution as a consequence of the urban environment constraints, described in section II. In the next step, four different techniques are employed for each specific error source.

Firstly, the video Fisheye camera has been chosen to detect GNSS satellite masking and exclude the corresponding measurements from the position solution. Secondly, a simple and efficient multipath mitigation technique will be utilized to mitigate the multipath at the GNSS receiver correlator output. Then, Vector Delay/Frequency Lock Loop (VDFLL) is implemented as a robust technique using a centralized approach to conduct joint signal tracking and position determination. In parallel, GNSS/MEMS hybridization is performed for measurement redundancy and consistency checking, knowing that inertial low-cost MEMS are capable of providing an independent position from the GNSS system and propagation channel.

The novelty of our approach consists in the integrity monitoring provision of a vectorized position solution, output of the VDFLL navigation filter. To provide a measure of trust on the position solution, two integrity monitoring techniques are taken into consideration, namely: the Receiver Autonomous Integrity Monitoring (RAIM) algorithm and Isotropy-Based Protection Level (IBPL) technique.

The core of our proposed algorithm is the VDFLL tracking loop, able to tackle both multipath errors and incongruent GNSS pseudo-range measurements. A more detailed description of each technique, proposed to mitigate the urban environment problematic, will be given in the following sections.

\section{VECTOR DELAY/FREQUENCY LOCK LOOP (VDFLL)}

Vector Tracking algorithm, first introduced in [7], represents an advanced GNSS signal processing technique due to its high performance in lower carrier-to-noise power density $\left(\mathrm{C} / \mathrm{N}_{\mathrm{o}}\right)$ ratios and in higher dynamics scenarios w.r.t. traditional GNSS tracking loops [8].

NLOS pseudo-range measurements and multipath errors are a direct consequence of urban environment. Therefore, traditional GNSS signal tracking techniques, which track the GNSS signals independently (scalar mode), sometimes fail to cope with these severe cases as tracking is possible. This is the reason why, in our approach, we propose the implementation of a VDFLL, capable of performing a joint GNSS signal tracking. In [7], it is stated that the vector tracking approach exploits the coupling between the receiver's dynamics and the dynamics seen by the tracking loops. Moreover in VDFLL, the signal tracking and navigation solution tasks are combined in a single step through the use of Least Square (LS) or Extended Kalman Filter (EKF) to simultaneously track the PRN code delays and the carrier frequencies.

Fig. 2 illustrates the block diagram of the VDFLL tracking and navigation filter implementation. For each channel 1 to $\mathrm{M}$, the 


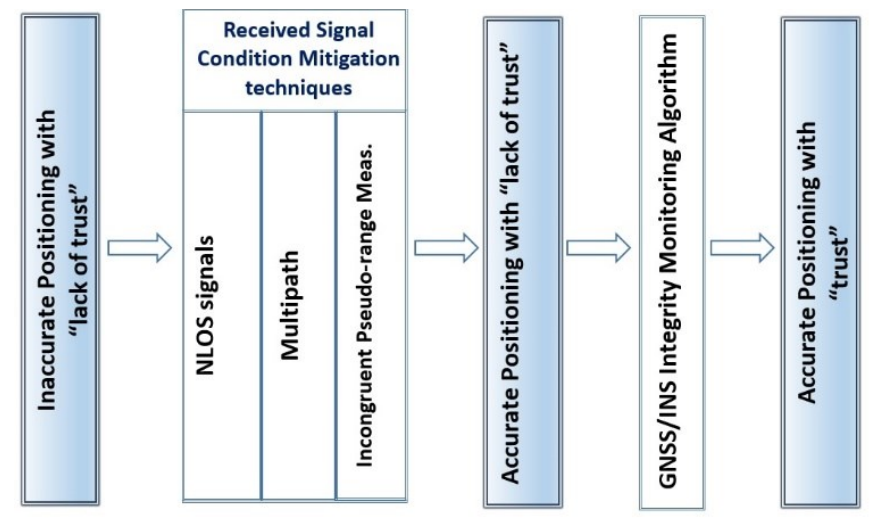

Figure 1. Schematic structure of the proposed step-by-step GNSS Integrity monitoring technique in dense urban environment.

pseudo-range and pseudo-range-rate discriminators output, denoted by $\Delta \rho$ and $\Delta \dot{\rho}$-, respectively, are fed to the central navigation $(\mathrm{EKF})$ filter.

The feedback to the code and carrier Numerical Control Oscillators (NCOs), for the two given configurations, is obtained from the EKF-computed pseudo-ranges and pseudo-range rates based on the most recent receiver's position and velocity estimate.

In the proposed algorithm, the chosen implementation is the position-state formulation [8] due to its feasibility for further extension of VDFLL to ultra-tight (deep) integration GNSS/MEMS. This can be achieved by replacing the navigation filter with an integrated GNSS/INS filter and also adding the MEMS error states.

The advantages and drawbacks of VDFLL technique with respect to scalar tracking are summarized in Table 1.

In the tight and ultra-tight coupled integration, the vector states will consist of inertial sensor (in our case MEMS) position, velocity error states along with GNSS clock offset and drift biases. The performance assessment of VDFLL implementation in ultra-tight coupling was investigated in [9].

\section{MICRO-MACHINED ELECTRO-MECHANICAL SYSTEM (MEMS) SENSORS}

In challenging environments, such as urban canyons, GNSS receivers suffer from the reception of incongruent-, multipathand interference-contaminated GNSS measurements. Therefore, our proposed solution relies on the integration of the GNSS system with the MEMS sensors, capable of supplying an independent position from GNSS systems and their propagation channels.

In the past years, MEMS have been rapidly developing to be the most widely used INS sensors for positioning and navigation due to their small size (in order of $\mathrm{cm}$ ), reduced production cost (price from $10 \$$ ), related to the silicon fabrication process. MEMS sensors are provided in different formats, such as: MEMS accelerometers (measuring the specific force $f$ of the vehicle along its sensitive axis), MEMS gyroscopes (measuring the body rotational motion $\omega$, across each sensitive axis, w.r.t. the inertial reference frame) or MEMS Inertial Measurement Unit (IMU) containing both 2 - or 3-axes accelerometers and

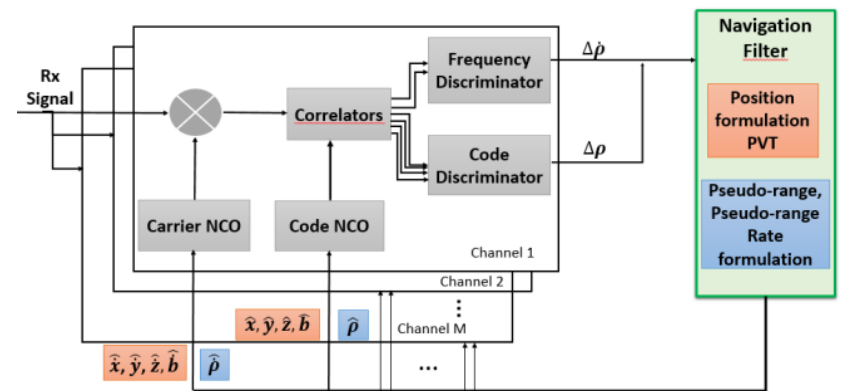

Figure 2. Block diagram of VDFLL tracking and navigation filter implementation with two possible configurations of the EKF filter.

gyroscopes along with the magnetometers measuring the heading of the vehicle. Therefore, a standalone position from the MEMS sensor can be provided from the use of two gyroscopes and one accelerometer. This will be reflected in a simplification of the EKF state vector, containing less bias states.

MEMS' low performance, as investigated in [10], makes them unsuitable to be used autonomously as a primary mean for positioning and navigation. Therefore, GNSS/MEMS integration yields many benefits, due to the complementary nature of these two systems, expressed in the following terms:

- MEMS are characterized by a short-term accuracy while on the other hand, GNSS system provides long-term positioning accuracy;

- In contrast to GNSS, MEMS performance is not affected from the surrounding environment and propagation channel;

- Contrary to the GNSS system, MEMS INS can be considered as an "offline" system immune toward interference and jamming.

In the framework of GNSS/MEMS hybridization, MEMS errors characterization is of great interest. This is related to the fact that the inertial sensor biases will be integrated in the state vector of the Kalman filter and are a necessary requirement for the integrity monitoring algorithm.

The single-axis MEMS gyroscope measurement models, consisting of both deterministic and random errors components, is expressed in the following form [11]:

$$
\begin{aligned}
\widetilde{\omega}=\omega & +b_{\text {cons }}+b_{\text {run-to-run }}+b_{\text {in-run }} \\
& +\left(S_{\text {cons }}+S_{\text {random }}\right) \omega+N \omega+\varepsilon(\omega),
\end{aligned}
$$

where: $\widetilde{\omega}$ denotes the single-axis gyroscope measurement; $\omega$ denotes the gyroscope true angular rate in $(\% / s) ; b_{\text {cons }}$ is the constant portion of the bias removed by the calibration process in units of $(\%) ; b_{\text {run-to-run }}$ is a deterministic bias express in $(o / s)$, slightly increasing its value every time the sensor is switched on; $b_{i n-r u n}$ is a stochastic bias occurring due to the changing environment conditions and in mostly modelled as a Gauss Markov process noise; $S_{\text {cons }}$ and $S_{\text {random }}$ are the constant and random portions of the MEMS gyroscope scale factor errors, which are the ratio of a change in output to the change in the intended input to be measured. 
TABLE I. VDFLL PERFORMANCE CHARACTERISTICS W.R.T. SCALAR TRACKING TECHNIQUE

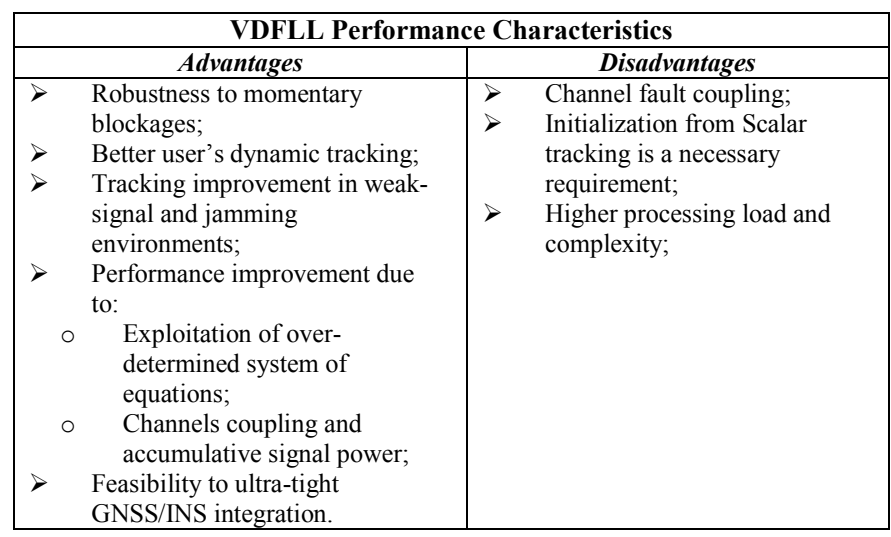

The deterministic part of the scale factor bias is mathematically denoted by the vector $\boldsymbol{S}_{\text {const }}=\left(s_{\text {const }, x}, s_{\text {const }, y}, s_{\text {const }, z}\right)$ expressed in parts per million (ppm) or \% unit; $N$ representing the non-orthogonalities or cross-misalignment errors, expressed in ppm or $\% ; \varepsilon(\omega)$ is a stochastic bias representing the MEMS sensor noise and its modeling is strictly datasheet related (e.g. Random Walk, First-Order Gauss Markov noise etc).

Similarly, the MEMS accelerometer measurement model, is given by [10]:

$$
\begin{gathered}
\tilde{f}=f+b_{\text {cons }}+b_{\text {random }}+S_{2} f^{2} \\
+\left(S_{\text {cons }}+S_{\text {random }}\right) \omega+N f+\delta g+\varepsilon(\omega),
\end{gathered}
$$

where: $\tilde{f}$ denotes the single-axis accelerometer measurement; $f$ denotes the accelerometer true specific force in $\left(\mathrm{m} / \mathrm{s}^{2}\right)$.

The different bias terms w.r.t. the gyroscope error model in Eq. (1) are the following: $b_{\text {random }}$ is the remaining random portion of the bias modelled as a Random Walk noise; $S_{2}$ denotes the nonlinear scale factor bias in (ppm or \%), being referred to as the anisoelastic bias (or $g^{2}$-dependent bias) expressed in $\left(\mathrm{m} / \mathrm{s}^{2} / \mathrm{g}^{2}\right)$ and has a minor contribution to the overall accelerometer bias; $\delta g$ denotes the anomalous gravity deviation from its theoretical value, expressed in $\left(\mathrm{m} / \mathrm{s}^{2}\right)$.

Recalling the MEMS sensor measurement model given in (1) and (2), it is possible to classify MEMS error contribution based on their nature in deterministic and random biases. This classification is helpful for a better understanding of the possible types of MEMS errors affecting our measurements and in order to identify the best procedures or models to minimize and preferably remove their contributions to the position solution, as summarized in Table II.

\section{PROPOSED GNSS NLOS REJECTION TECHNIQUE}

In dense urban environments, a frequently occurring phenomenon, denoted in this paper as only NLOS signals reception, is the lack of reception of the LOS GNSS signal combined with the reception of only NLOS GNSS signals. As a consequence, a degraded position accuracy is observed due to the introduced error on the pseudo-range measurements.

The strategy proposed in this paper to overcome this phenomenon, consists in using a Video Fisheye camera to perform GNSS NLOS rejection and discard the erroneous pseudo-range measurements at the source with the main goal of achieving better GNSS/INS integrity monitoring. The Fisheye camera, characterized by a large field of view (typically $180^{\circ}$ ), is mounted on the vehicle roof and oriented upwards to capture the sky images while the vehicle is moving along its trajectory.

The Fisheye camera technique, for GNSS satellite detection, was firstly proposed in [12] and is based on sequential image processing steps, as illustrated in Fig. 3. The starting point of the Fisheye technique is the image data acquisition from the Fisheye camera with a frame rate of 60 frames per second (fps). Once the data have been acquired, the image processing phase is initiated consisting of four successive steps [12]:

- Image simplification: using a geodesic reconstruction by dilatation (RGD) with an optimum parameter of contrast $\mathrm{H}$, as specified in [12];

- Pixels classification in two classes: sky and not sky (represented by buildings, vegetation, lampposts etc), by using image clustering classification algorithms [12];

- Morphological post-treatment: consists in the reclassification of the uncertainty regions.

- Evaluation: where the performance assessment of the three previous image processing techniques is carried out. In this step the image processing computational time is elapsed, as a crucial indicator for real-time

\begin{tabular}{|c|c|c|c|c|c|c|}
\hline \multirow[t]{2}{*}{ MEMS Errors (Biases) } & \multirow[t]{2}{*}{ Symbol } & \multirow[t]{2}{*}{ Unit } & \multicolumn{2}{|c|}{ Errors Nature } & \multicolumn{2}{|c|}{ Minimization or Removal procedures } \\
\hline & & & Deterministic & Random & Calibration & Stochastic Modeling \\
\hline Constant bias & $b_{\text {cons }}$ & $\begin{array}{c}\text { Gyro: } \% / \mathrm{s} \\
\text { Accelero: } \mathrm{m} / \mathrm{s}^{2}\end{array}$ & $\mathbf{x}$ & & $\mathbf{x}$ & \\
\hline Run-to-run bias & $b_{\text {run-to-run }}$ & $\begin{array}{c}\text { Gyro: } \% / \mathrm{s} \\
\text { Accelero: } \mathrm{m} / \mathrm{s}^{2}\end{array}$ & $\mathbf{x}$ & & $\mathbf{x}$ & \\
\hline In-Run bias stability & $b_{\text {in-run }}$ & $\begin{array}{c}1 \sigma \text { value } \\
\text { Gyro: } \% / \mathrm{s} \\
\text { Accelero: } \mathrm{m} / \mathrm{s}^{2}\end{array}$ & & $\mathbf{x}$ & & $\mathbf{x}$ \\
\hline \multirow[t]{2}{*}{ Scale - factor bias } & $S_{\text {cons }}$ & ppm or $\%$ & $\mathbf{x}$ & & $\mathbf{x}$ & \\
\hline & $S_{\text {random }}$ & $1 \sigma$ value & & $\mathbf{x}$ (negligible) & & $\mathbf{x}$ \\
\hline $\begin{array}{l}\text { Non-orthogonalities or } \\
\text { cross-misalignment bias }\end{array}$ & $N$ & ppm or $\%$ & $\mathbf{x}$ & & $\mathbf{x}$ & \\
\hline Anisolastic bias & $S_{2}$ & $\mathrm{~m} / \mathrm{s}^{2} / \mathrm{g}^{2}$ & $\mathbf{x}$ & & $\mathbf{x}$ & \\
\hline Gravity deviation & $\delta g$ & $\mathrm{~m} / \mathrm{s}^{2}$ & $\mathbf{x}$ & & $\mathbf{x}$ & \\
\hline Sensor Noise & $\varepsilon$ & $1 \sigma$ value & & $\mathbf{x}$ & & $\mathbf{x}$ \\
\hline
\end{tabular}
applications. 


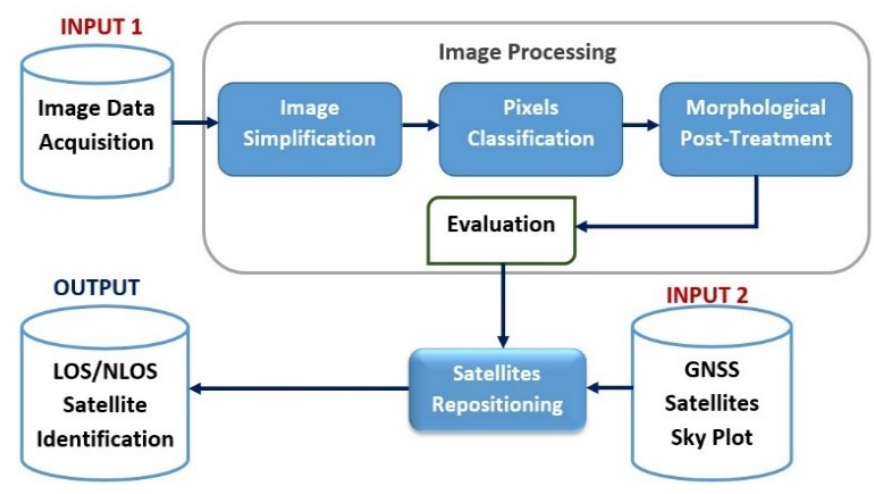

Figure 3. Block diagram of LOS GNSS satellites detection using the Fisheye camera.

The crucial point of the Fisheye technique is the GNSS satellites repositioning, performed through the overlapping of the satellites sky plot from the GNSS receiver on board of the vehicle, containing their positions in terms of azimuth and elevation angles and the simplified Fisheye image.

Finally, the output of this algorithm is the identification of the GNSS satellites located in the non-sky region, which will further be excluded from the position calculation.

\section{Multipath Mitigation TechniQUe}

Multipath is the dominant cause of pseudo-range errors for GNSS systems in urban environment and is defined as the echoes generated from the reflection and diffraction of the LOS GNSS signal on the environment obstacles. From the receiver's point of view, the main multipath effect is the distortion of the correlation function between the received signal and the receiver's generated local replica. This will lead to a significant error first in the satellite pseudo-range measurement which will propagate to the receiver's position estimate.

The mostly used multipath mitigation approaches rely on the GNSS code correlation techniques based on the DLL estimators. An extensive list of several correlation-based multipath mitigation techniques is given in [13]. However, the fact that the multipath impact on the signal tracking performance is strictly dependent on the used tracking technique and also on the signal modulation should be underlined [15]. As previously described in Section III, the main part of the proposed algorithm consists in the implementation of VDFLL, which by nature can mitigate part of the multipath effects due to the information sharing between the different tracking channels.

To emphasize the multipath resistance of the VDFLL, it is then necessary to feed inputs to the VDFLL that are as multipath-free as possible. Having this in mind, the choice was oriented toward the use of the High Resolution Correlator (HRC) discriminator, part of the Double-Delta $(\Delta \Delta)$ category of discriminator-based DLL techniques that was firstly presented in [15]. Its code discriminator function is set up through the linear combination of 5 correlators, such as: Early (E), Late (L), Prompt (P), Very-Early (VE) and Very-Late (VL) correlators. Through this linear combination of the (E-L) with a spacing equal to $d$ chips and the (VE-VL) couple having a spacing of $2 d$ chips, a much narrower discriminator function w.r.t. the traditional chip pulse shaping autocorrelation function is achieved as [15]:
Or,

$$
D_{H R C}=\left(E_{1}-L_{1}\right)-\frac{1}{2}\left(E_{2}-L_{2}\right)
$$

$$
D_{H R C}=\operatorname{Narrow}(d)-\operatorname{Narrow} \frac{1}{2}(2 d),
$$

where typically the spacing $d=0.05$ chips.

The drawbacks of such techniques are that they requires a large receiver bandwidth and are patented.

\section{INTEGRITY MONITORING TECHNIQUE}

In the previous sections, different techniques were described as capable of overcoming or mitigating the problems of GNSS positioning in dense urban environments. However, this approach manages only to provide a more accurate positioning but still does not ensure a measure of trust of the navigation solution. Therefore, to obtain a reliable position solution answering the application requirements, the implementation of an integrity monitoring technique is a necessary requirement.

In this paper, two potential candidate techniques providing integrity monitoring of the position solution, namely, RAIM and Isotropy-Based Protection Level (IBPL), will be analyzed. However, both these models do not yet fully address the multipath fault modeling in urban environment.

\section{A. Receiver Autonomous Integrity Monitoring (RAIM) technique}

RAIM algorithms, widely employed in the civil aviation domain, are measurement rejection techniques that are run autonomously by the GNSS receiver. In RAIM, first the Fault Detection and Exclusion (FDE) mechanism is executed and followed by the Protection Level (PL) computation, by taking into account only the GNSS measurements that passed the FDE test. The main advantage of RAIM-based algorithms, is the provision of tight protection levels with respect to IBPL, with the requirement of six pseudo-range measurements to compute the FDE procedure. The adaptation of the classic RAIM techniques for hybridized GNSS/INS systems and to protect against multiple failures, is presented in [16].

\section{B. Isotropy-Based Protection Level (IBPL) technique}

IBPL, presented in [17], is specifically developed for the Liability Critical land applications and quietly differs from the other RAIM-based integrity techniques, due to the fact that no measurement rejection is performed. Therefore, the implementation of Isotropy-Based Protection Level (IBPL) algorithm, due to the only requirement of four GNSS measurements (six measurements in RAIM) to provide the protection level computation, is proposed.

In IBPL, the protection level is estimated from the vector of the pseudo-range residuals, The Horizontal Protection Level (HPL), depending linearly on the size of the pseudo-range residual vector $\delta \boldsymbol{\rho}(k)=\left[\delta \rho^{i}(k)\right], i=1 \ldots N$ and the state estimation error $\delta \boldsymbol{x}_{\mathrm{i}, 3 \times 1}$, is computed as follows [17]:

$$
H P L=k \cdot\|r\| \cdot H D O P,
$$

where: $r$ denotes the least square residual vector; HDOP is the horizontal dilution of precision and $k$ is called the isotropy confidence ratio (ICR), which is defined in [16] to ensure the 
state estimation error $\delta$ is bounded by the size of the residual vector up to the ICR and the target confidence level $1-\alpha$ [17]:

$$
P(\|H \cdot \delta\|>k \cdot\|r\|) \leq \alpha,
$$

where: $H$ is the least square observation matrix and $\alpha$ is the integrity risk value, specified in the application requirements. ICR can be computed using multidimensional integral equations dependent on the integrity risk value $\alpha$ and the number of measurements $N$, as follows:

$$
k=k(\alpha, N) .
$$

The main drawback of this technique is related to the large protection levels (PL), because no exclusion of erroneous GNSS pseudo-range measurements is performed.

\section{CONCLUSIONS}

In this work, an GNSS integrity monitoring approach of an integrated GNSS/MEMS system aided by the Video Fisheye camera was presented. The specific implementation of our stepby-step approach able to cope with the major GNSS positioning error sources in urban canyons has been discussed.

In specific, this paper analyzed the formulation of the Vector Delay Frequency Lock Loop (VDFLL), as the chosen advanced tracking technique capable of coping with incongruent measurements and also capable of providing multipath mitigation. Moreover, VDFLL advantages and disadvantages w.r.t. to the classical scalar tracking techniques were highlighted.

Afterwards, MEMS error characterization was discussed, since they will be integrated in the state vector of the Kalman filter while the MEMS stochastic error components are required for the integrity protection level computation. Following, the proposed use of the Video Fisheye camera as a NLOS GNSS satellite detection was analyzed. This technique exhibits a high potential for an increased positioning accuracy by excluding the NLOS satellites prior to the navigation solution. Later on, the possible implementation of a simple and effective multipath mitigation technique, such as HRC, was also assessed.

Finally, two candidates for the integrity monitoring provision in urban environments, were discussed.

\section{FUTURE WORK}

Ongoing work is focused toward the definition and analysis of target urban applications and their associated user requirements. Of great interest is the exploitation of GNSS/MEMS hybridization techniques along with the definition of the Measurement Quality Indicators related to the specific choice of the inertial sensors. Our main focuses will be dedicated first to implement the GNSS/MEMS hybridization + video fisheye algorithms and second to the determination of the novel integrity monitoring algorithm that is well suited to the tracking algorithm, measurements' models (in nominal and failure modes) and chosen hybridization technique.

In the framework of this project, our research team is currently in the designing process of a test vehicle equipped with high performance tactical-grade INS system along with a high quality GNSS multi-constellation Novatel receiver for the reference trajectory and a camera system, containing the Fisheye camera and optical lens. Therefore, real-life experimentation of our positioning algorithm and integrity monitoring technique will be carried out in urban campaigns in Toulouse.

\section{ACKNOWLEDGMENT}

This work was financially supported by EU FP7 Marie Curie Initial Training Network MULTI-POS (Multi-technology Positioning Professionals) under grant nr. 316528

\section{REFERENCES}

[1] S. G. Lanza, C. B. Gutierrez and J. Cosmen-Schortmann, "GINA-GNSS for innovative road applications: EGNOS / Galileo for Road User Charging and Value Added Services," Intelligent Transport Systems Telecommunications (ITST), IEEE, pp: 348-352, 20-22 Oct. 2009.

[2] K. Sheridan, D. Wells, C. Botteron, J. Leclère, F. Dominici and A Defina, "An Assisted-GNSS Solution for Demanding Road Applications using the EGNOS Data Access System (EDAS)," Proceedings of the European Navigation Conference on GNSS, ENC-GNSS, 2010.

[3] 'Discussion paper - Intelligent Roads: What can the Road community expect from GALILEO?", European Union Road Federation (ERF), Brussel Programme Centre, April 2006.

[4] U. I. Bhatti and W. Y. Ochieng, "Detecting Multiple failures in GPS/INS integrated system: A Novel architecture for Integrity Monitoring," Journal of Global Positioning, vol.8, No.1, pp: 26-42, 2009

[5] Z. Jiang, P. D. Groves, W. Y. Ochieng, S. Feng, C. D. Milner and P. G. Mattos, "Multi-Constellation GNSS Multipath Mitigation Using Consistency Checking," Proceedings of the 24th ION-ITM GNSS, pp. 3889 - 3902, Portland OR, September 19-23, 2011.

[6] Elliott D. Kaplan, Christopher J. Hegarty, "Understanding GPS: Principles and Application," $2^{\text {nd }}$ edition, Artech House, 2006.

[7] J. J. Spilker Jr., "Fundamentals of signal tracking theory," in Global Positioning System: Theory and Applications, Volume 1, 1996.

[8] M. Lashely., "Modeling and performance analysis of GPS vector tracking algorithms", Ph.D Thesis, Auburn Unibversity, 2009

[9] M.G. Petovello, G. Lachapelle, "Comparison of Vector-Based Software Receiver Implementations with Application to Ultra-Tight GPS/INS Integration". ION GNSS 2006, Fort Worth, TX, USA, September 2006.

[10] D. H. Titterton and J. L. Weston, Strapdown Inertial Navigation Technology, 2nd ed., Stevenage, U.K, IEEE, 2004.

[11] P. Aggarwal, Z. Syed, A. Noureldin, N. El-Shemy, MEMS-Based Integrated Navigation, Artech House, ISBN: 978-1-60807-043-5 2010 .

[12] D. Attia, C. Meurie, Y. Ruichek, J. Marais, A. Flancquart, "Image analysis based real time detection of satellites reception state", 13th International IEEE Annual Conference on Intelligent Transportation Systems, Madeira Island, Portugal, September 19-22, 2010.

[13] M. Zahidul, H. Bhuiyan and E. S. Lohan, “Advanced Multipath Mitigation Techniques for Satellite-Based Positioning Applications", International Journal of Navigation and Observation Volume 2010 Article ID 412393

[14] O. Julien, "Design of Galileo L1F Receiver Tracking Loops", $P h D$ Thesis, published as UCGE Report No. 20227, Department of Geomatics Engineering, The University of Calgary, 2005.

[15] G. A. McGraw and M. S. Braasch, "GNSS Multipath Mitigation Using Gated and High Resolution Correlator Concepts," NTM 1999, San Diego, CA, January 1999.

[16] J. Diesel, G. Dunn, "GPS/IRS AIME: Certification for Sole Means and Solution to RF Interference," ION GPS 1996, Kansas City, MO September 1996.

[17] J. Cosmen-Scortmann, M. Azaola-Saenz, A.M Martinez-Olague and M. Toledo-Lopez, "Integrity in urban and road environments and its use in liability critical applications," Position, Location and Navigation Symposium (PLANS), IEEE/ION, pp: 972-983, 5-8 May 2008 